\title{
INTERALLELIC COMPLEMENTATION AT THE ad-2 LOCUS OF SACCHAROMYCES CEREVISIIE
}

\author{
R. A. WOODS \\ Department of Genetics, University of Sheffield \\ and \\ E. A. BEVAN \\ Department of Botany, Queen Mary College, London
}

Received 20.vi.65

\section{INTRODUCTION}

INTERALLELIC complementation has been observed at loci concerned with purine synthesis in Aspergillus nidulans (Calef, 1956); Neurospora crassa (Giles, Partridge and Nelson, I957; Giles, I958; Ishikawa, I96o; de Serres, 1963); Schizosaccharomyces pombe (Megnet, 1959; Leupold, I96 I); Salmonella typhimurium (Gots, Goon and Hild, r 959); and Saccharomyces cerevisia (Bevan and Woods, I962; Costello and Bevan, I964; Dorfmann, 1964).

Mutants at the $a d-I$ and ad-2 gene loci of Saccharomyces cerevisia accumulate intracellular red pigments which have been identified as aminoimidazole ribonucleotide $(a d-I)$ and aminoimidazole carboxylic acid ribonucleotide ( $a d-2)$ (Levinthal, Fogel and Hurst, 1963). The two loci are on different chromosomes and are functionally independent; diploids of the constitution $a d-I^{+}, a d-2 / a d-I, a d-2^{+}$are white and prototrophic.

Independently isolated mutants at both loci have been tested for interallelic complementation. None was found between those at the ad-I locus (Bevan and Woods, I962; Bevan and Makower, unpublished). In this report data are presented showing that the pattern of complementation between mutants of the ad-2 locus is complex; the complementation map constructed from the results of tests on 150 mutants is composed of three sections, two of which are circular and the other linear.

\section{MATERIALS AND METHODS}

The complete, minimal and sporulation media used were those listed by Cox and Bevan (1962).

The standard $a d-I$ and $a d-2$ alleles were derived from strains $99 \mathrm{R}$ (Reaume and Tatum, 1949) and 276/3br (Ephrussi, Hottinguer and Tavlitski, 1949). Wild-type strains $W_{4}, W_{5}, Y_{5}$ ob and $F_{1}$ were obtained from the Oxford stock collection. The remainder of the $q d-I$ and $a d-2$ mutants were isolated as red sectors or whole colonies from platings of either wild-type haploid cells, or ascospores, treated with ultra-violet irradiation. The mutants fall into two series. Firstly, strains $\mathrm{RI}_{\mathrm{I}}$ to R222 were isolated following irradiation of a liquid paraffin suspension of ascospores (cf. Emeis and Gutz, I958) from a WT $4 \times \mathrm{WT}_{5}$ cross. Secondly, strains ad-2.I to ad-2.22 were obtained from the irradiation of strains $Y_{5} \mathrm{ob}, \mathrm{F}_{\mathrm{I}}$ and $\mathrm{WT}_{4}$. To 
ensure genetic homogeneity these mutants were crossed with $\mathrm{WT}_{4}$ or $\mathrm{WT}_{5}$ wild type haploids and reisolated.

In order (i) to distinguish the $a d-I$ from the $a d-2$ mutants and (ii) to determine their mating types, each mutant strain was replica-plated (Lederberg and Lederberg, 1952) on to plates of complete agar medium already spread with lawns of cells of each of the mating types of both standard strains, viz. $a d-I \alpha, a d-I a, a d-2 \alpha$ and ad $2 a$. White diploid cells resulted only when the background cells were both nonallelic and of opposite mating type to the replicated cells. This test depended on the fact that neither of the standard tester strains has ever shown interallelic complementation.

Stock cultures of each of the ad-2 mutant strains were obtained in both mating types by crossing them to either the wild-type $\mathrm{WT}_{4}$ or $\mathrm{WT}_{5}$ haploid strains and selecting the appropriate ascospore cultures from the resulting diploid.

Each ad-2 strain was tested for complementation with the others as follows: master plates each containing 30 different $a d-2$ strains and one $a d-I$ strain all of $\alpha$ mating type were replicated on to a series of complete agar medium plates already spread with a background lawn of cells of strains of mating type $a$, including a plate of $a d-I$. Master plates were also replicated on to a plate of complete medium agar. All of these plates were incubated at $28^{\circ} \mathrm{C}$. for Io- 12 hours to allow for mating and the formation of diploid clones. Following this they were replicated on to plates of minimal agar medium and incubated for four days. Two criteria were adopted for showing that complementation had occurred between a pair of strains: (i) growth of a white diploid on complete medium, and (ii) the non-requirement for adenine and therefore growth of the diploid on minimal medium. The presence of the ad-I strain on the master and background plates allowed the immediate detection of any mating anomaly among the strains used. A comparison of the complete medium plate and its replica on minimal medium provided information on the leakiness or reversion of the strains on the master plates.

It should be noted that each pairwise combination of mutant alleles was tested twice in the course of the main series of experiments since reciprocal tests involving haploid strains were carried out, e.g. ad-2.I $a \times a d-2 \cdot 2 \alpha$ and $a d-2 \cdot I \alpha \times a d-2 \cdot 2 a$. Doubtful and anomalous results were retested by mating single cells of the strains concerned and comparing the growth of the resulting diploid with that of each of the parental haploid strains.

Both single-cell matings and ascus dissection were carried out by micromanipulation on open plates (Kemp and Bevan, 1959). Prior to dissection, asci were treated with digestive juice from Helix aspersa to soften their walls (Johnston and Mortimer, 1959).

\section{RESULTS}

Out of the total of 240 pigmented adenine auxotrophs 78 proved to be mutant at the $a d-I$ locus and $\mathrm{I}_{2}$ at the $a d-2$ locus. Tests for complementation were confined to I5O of the available ad-2 mutants and these were classified initially either as non-complementing, of which there were 56 , or complementing, of which there were 94. Of the latter, $5^{\mathrm{I}}$ proved to be non-leaky and 43 leaky. Only one of the non-complementing mutants $(a d-2 \cdot 3)$ was leaky.

Thirty of the leaky mutants could be scored satisfactorily in all the tests and are included in the matrix and maps together with the nonleaky mutants. The designation of these in the order in which they occur in the matrix (fig. I) is as follows: $R_{I}{ }_{10}, R_{I}, R_{67}, R_{I}{ }_{3}, R_{I} 8 I$, ad-2.4, R I I 7, ad-2.15, R I 16, R I62, R I67, R36, R82, R26, R I84, R27,

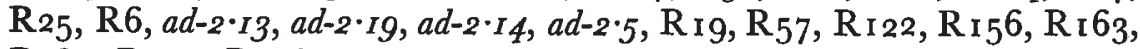
Ri89, Ri 72, Rig8. 


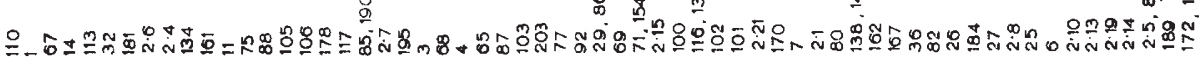
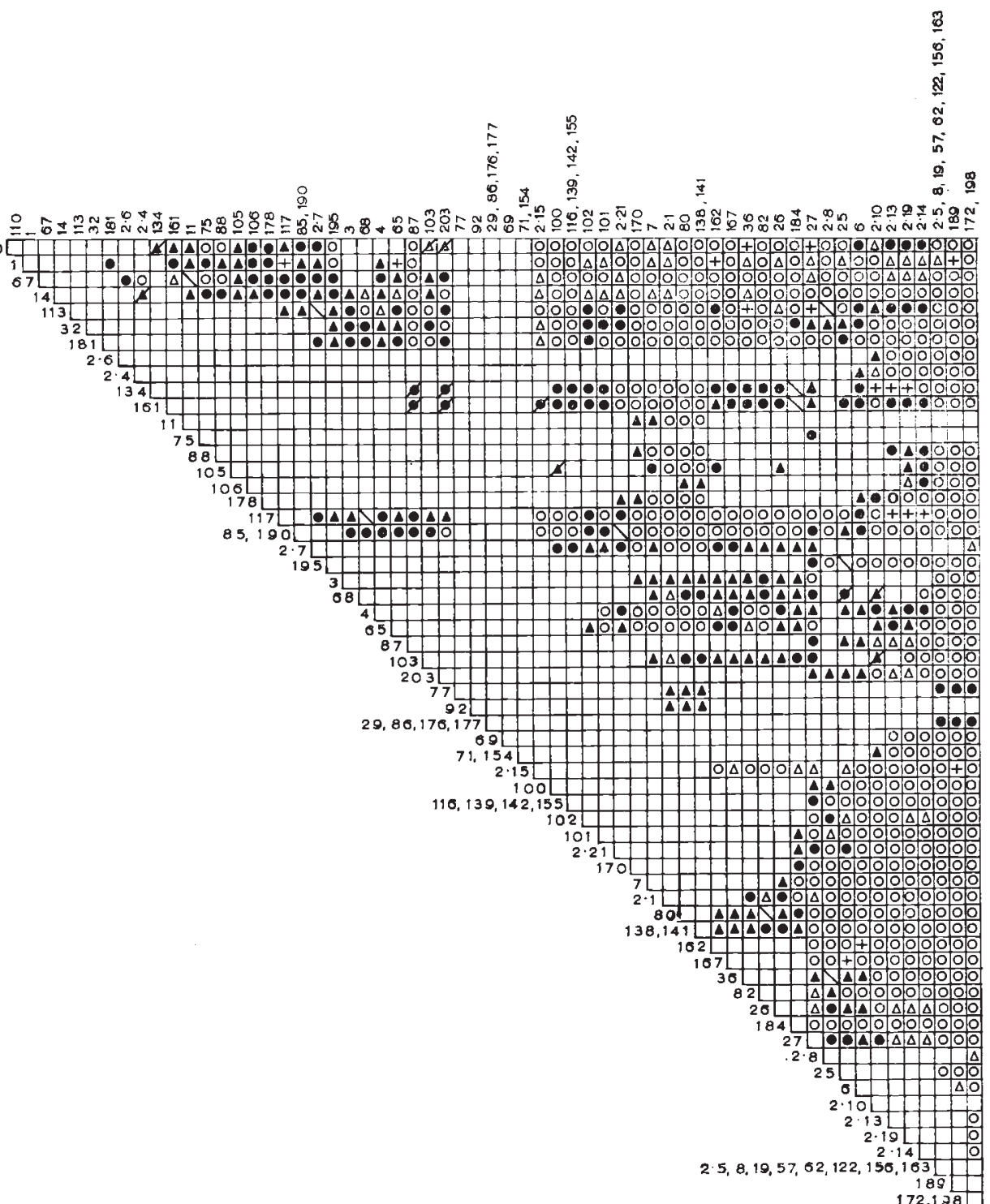

Fig. 1.-Matrix of results for 78 complementing ad-2 mutant alleles.

$\Delta$ Growth poor, pigmented.

- Growth good, pigmented.

$\triangle$ Growth poor, white.

O Growth good, white.

+ Result positive but contaminated or reverting.

Anomalous results: $\square$ Negative in matrix, positive in map (13).

The series prefixes of the mutants have been omitted. 
Eleven of the complementing mutants are omitted because they proved either to have high reversion rates or were too leaky to score with confidence in all the tests.

The matrix of results of 78 mutants is shown in fig. $\mathrm{r}$, and an interpretation of this matrix in the form of a linear complementation map is shown in fig. 2. The map is subdivided into three sections: Section A has 12 sub-units (r-12), Section B has 14 sub-units (13-26),

A

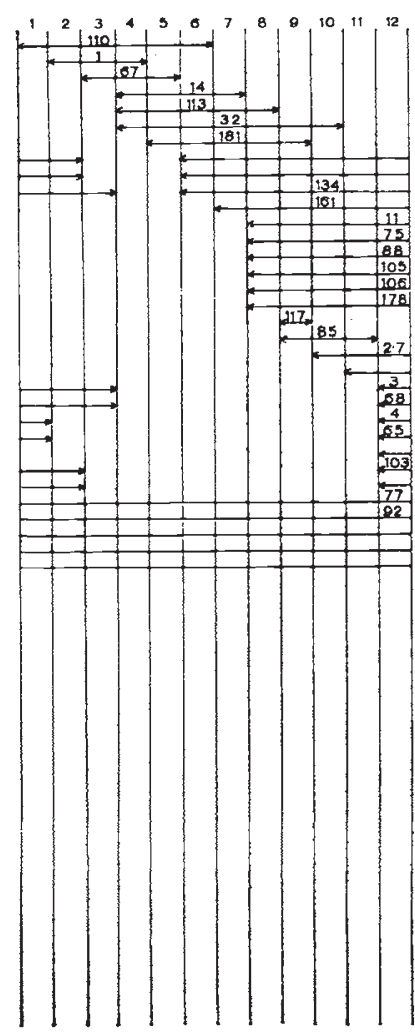

B

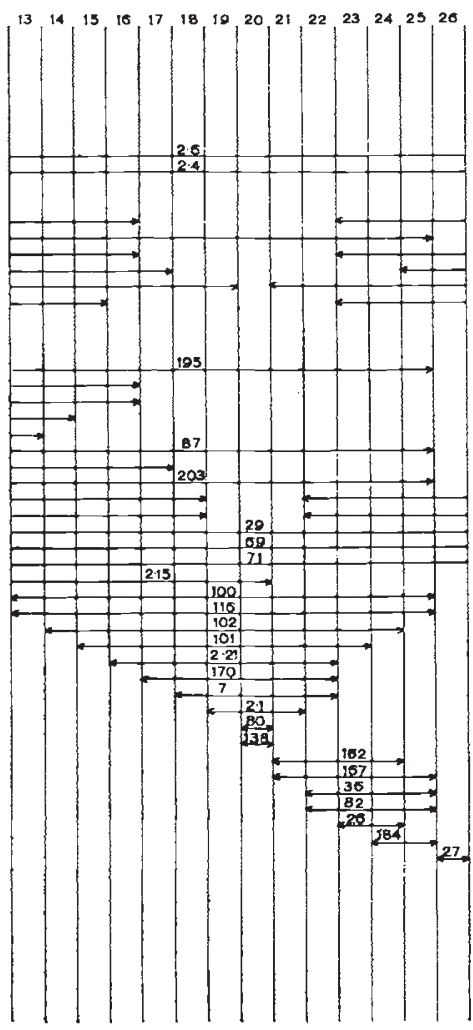

C

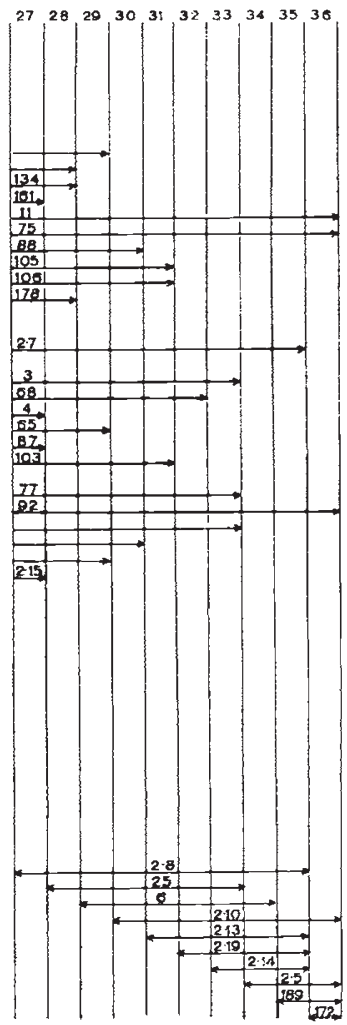

Fig. 2. - Linear representation of the complementation map of the ad-2 mutants. The three sections of the map are separated in the figure. The arrowheads indicate the limits of the individual mutants in the map.

and Section $\mathrm{C}$ has ro sub-units (27-36). In a conventional linear complementation map some of the mutants allotted to Sections $A$ and $B$ can only be represented by broken lines. Thus $R_{3}$ complements $R_{14}$, $R_{I I} 3$ and $R_{32}$ which are in the centre of Section $A$, but fails to complement other mutants which are placed to the left and right of these three. However, $R_{3}$ can be represented as an unbroken line if the ends of Section $A$ are joined to form a circular map. Similar considerations apply to some of the mutants allotted to Section B, e.g. 


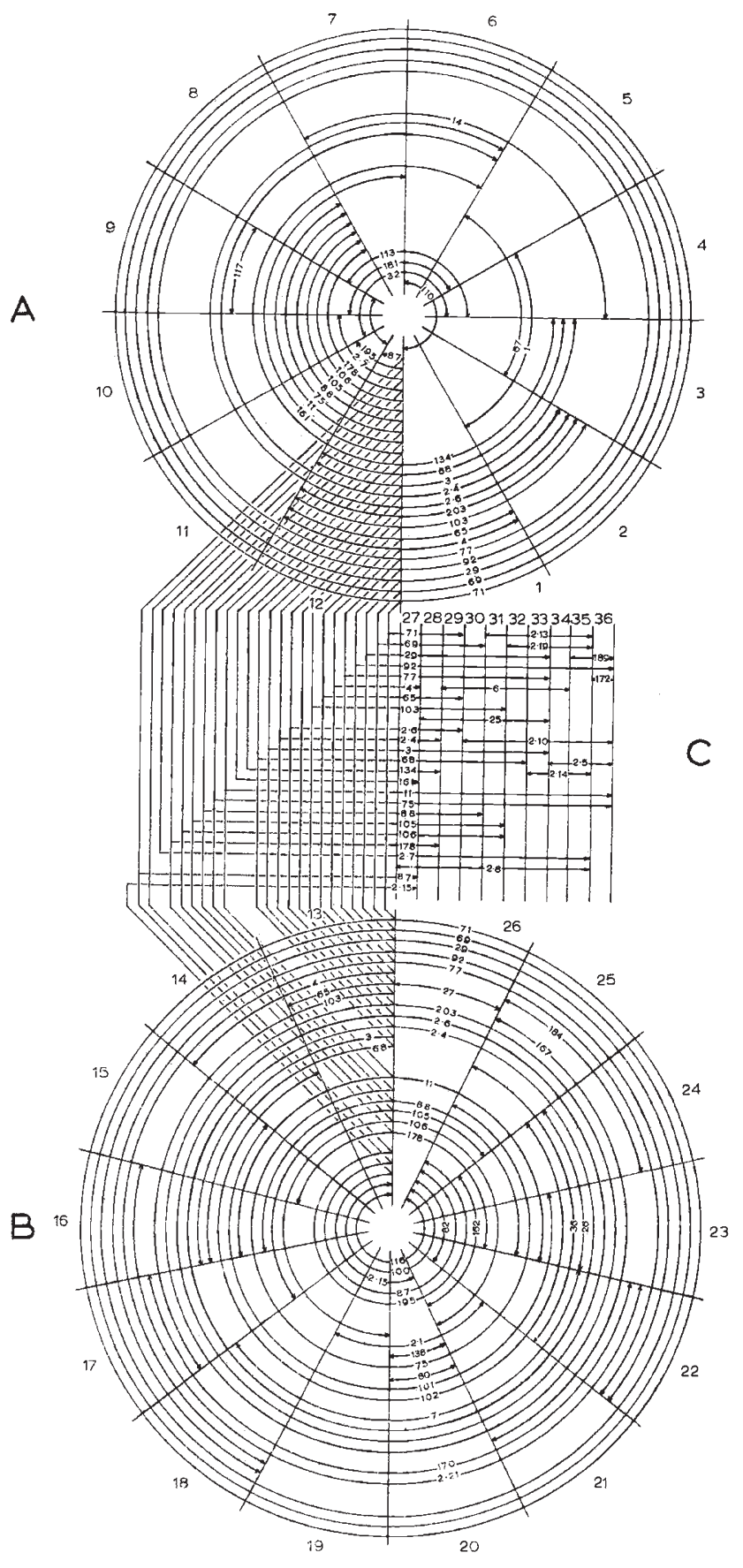

Fic. 3.-A more complicated map-form in which circularity has been achieved for Sections $A$ and $B$ while Section $\mathbf{C}$ is represented in a linear manner between them. 
$R_{I I}$. Therefore, both of these sections can be planned in a circular fashion, whereas Section $\mathrm{C}$ remains linear. Fig. 3 shows a more complex map form in which circularity for Sections A and B has been achieved, whilst Section $\mathrm{C}$ is shown as a linear structure between them.

Twenty-three anomalous complementation reactions involving a total of twenty-eight mutants are portrayed as either negative in the matrix and positive in the map or vice versa (see fig. I). Any other arrangements of these twenty-eight mutants would lead to more discrepancies between the matrix and the map. These anomalies do not appear to be more common in particular regions of the map (cf. Dorfman, 1964) and in several instances pairs of mutants with identical or very similar complementation patterns give the same anomalous result when tested with a third mutant, e.g. $\mathrm{R} 85$ and Rigo (identical) $\times a d-2 \cdot 21 ; \mathrm{R}_{\mathrm{I}} 34$ and $\mathrm{R}_{\mathrm{I}} 6 \mathrm{I}$ (similar) $\times \mathrm{R}_{77}, \mathrm{R}_{203}$ (similar) and $\mathrm{R}_{\mathrm{I}} 84$.

TABLE I

A comparison of the complementing ability of leaky and non-leaky mutants

\begin{tabular}{|lr|c|c|c|}
\hline \multicolumn{1}{|c|}{ Mutant combination } & \multicolumn{3}{|c|}{ Results } \\
\cline { 2 - 5 } & & Possible & Positive & $\begin{array}{c}\text { Positive } \\
\text { (per cent.) }\end{array}$ \\
\hline Leaky $\times$ leaky . & $\cdot$ & 420 & 310 & $73 \cdot 8$ \\
Leaky $\times$ non-leaky. & $\cdot$ & $144^{\circ}$ & 850 & $59^{\circ}$ \\
Non-leaky $\times$ non-leaky & $\cdot$ & 1104 & $33^{\circ}$ & $29 \cdot 9$ \\
\hline
\end{tabular}

Most of the anomalies concern mutants which just overlap or are separated by only one or two sub-units of the complementation map but two, R85 and Rigo $\times a d-2 \cdot 2 I$, and $\mathrm{R}_{100} \times \mathrm{R}_{105}$, involve mutants which, in the first instance are separated by five sub-units, and in the second, overlap for six sub-units of the map. It is, of course, possible that these anomalies would disappear if the complementation map were run into the ground.

Five leaky mutants, $a d-2 \cdot 2, R_{23}, R_{14} 6, R_{1} 75$ and $R_{192}$ could not be integrated into the proposed map. If included, they would cause a significant increase in the number of anomalous results.

Excluding homoallele tests there are 3003 pairwise combinations of the 78 mutants. Of these 1490 showed complementation. The average number of positive reactions per leaky mutant was $38 \cdot 6$ whereas the average for non-leaky mutants was $24 \cdot 6$. This difference between the two types of mutants is further illustrated in table $\mathrm{I}$.

The average number of map sub-units covered per leaky mutant is 4.6 compared with 14.3 for non-leaky mutants. The molecular defects in leaky mutants might be expected to be less extensive than 
those in non-leaky mutants. If this is so, they are expected to be involved in a greater proportion of positive complementation reactions.

There is some evidence for clustering of the leaky mutants in particular regions of the complementation map. Six of them (R I Io, $R_{1}, R_{67}, R_{113}, R_{18} I_{1}$ and $R_{I 17}$ ) are confined to sub-units $I$ to 9 of Section A; seven ( $R_{1} 6_{2}, R_{16} 6, R_{3} 6, R_{82}, R_{26}, R_{1} 8_{4}$ and $R_{27}$ ) to sub-units 21 to 26 of Section $B$, and fourteen (R25, R6, ad-2.I3,

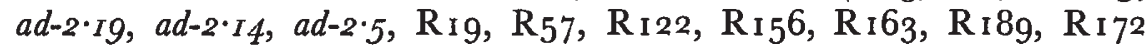
and $\left.R_{198}\right)$ to sub-units 28 to 36 . Of the remaining three mutants, ad-2.4 extends into all three sections, ad-2.15 into Sections $\mathrm{B}$ and $\mathrm{C}$, and RII 6 is confined to Section B.

\section{DISCUSSION}

Interallelic complementation is thought to be due to the interaction of differently defective polypeptides to give an aggregate with some activity. This hypothesis, initially proposed by Catcheside and Overton (1958), was elaborated by Brenner (1959) who suggested that

TABLE 2

Complementation at analogous loci concerned in adenine synthesis

\begin{tabular}{|c|c|c|c|}
\hline Organism & $\begin{array}{l}\text { Complementing } \\
\text { locus }\end{array}$ & $\begin{array}{c}\text { Non-complementing } \\
\text { locus }\end{array}$ & Reference \\
\hline $\begin{array}{l}\text { Salmonella } \\
\text { Neurospora } \\
\text { Saccharomyces } \\
\text { Schizosaccharomyces }\end{array}$ & $\begin{array}{l}a d-E \\
a d-3 B \\
a d-2 \\
a d-6\end{array}$ & $\begin{array}{l}a d-C \\
a d-3 A \\
a d-1 \\
a d-7\end{array}$ & $\begin{array}{c}\text { Gots, Coon and Hild } \\
\text { (1959) } \\
\text { de Serres } \\
\text { (1960, 1963) } \\
\text { Bevan and Woods } \\
\text { (1962) } \\
\text { Leupold (1961) }\end{array}$ \\
\hline
\end{tabular}

enzymes showing complementation might be normally polymeric and the constituent monomers identical. Thus the units involved in complementation were defective monomers which somehow compensated each other when polymerised. More detailed proposals concerning the molecular mechanism of complementation have since been made by Crick and Orgel (1964).

Experimental support for the polymer hypothesis is mainly drawn from studies on the alkaline phosphatase of Escherichia coli. This enzyme has been shown to be a dimer, composed of two apparently identical monomers (Levinthal, Signer and Fetherolf, 1962; Rothman and Byrne, 1963). Further, Schlesinger and Levinthal (1963) have shown that complementation between alkaline phosphataseless mutants involves the formation of hybrid dimers.

Similar conclusions have been reached concerning the mechanism of complementation at the am locus of Neurospora crassa. Complementing mutants form hybrid aggregates (Fincham and Coddington, 1963) 
and it seems probable that the enzyme is polymeric (Fincham and Stadler, i965).

However, detailed biochemical and biophysical analyses of complementation have been attempted in relatively few instances and for the majority of the loci at which it has been reported there are only complementation maps.

Initially complementation maps were all linear with end points, though exceptional mutants were noted. More recently the patterns of complementation at a number of loci have required the construction of non-linear, circular, or helical maps. A circular map allows considerably more latitude in mutant placement than a simple linear one with the same number of sub-units (Carlson, rg6r), consequently, it might be supposed that complementation maps have limited biological significance. This seems unlikely in view of the high degree of colinearity which has generally been found between genetic and complementation maps. For example, colinearity has been demonstrated for a linear map (Case and Giles, 1960) a circular map (Kapuler and Bernstein, 1963 ) and a map containing both circular and linear sections (Leupold and Gutz, 1964). It thus seems possible, as has been suggested by Kapuler and Bernstein (1963), that the complementation map of a locus may bear direct relationship to the tertiary structure of the enzyme sub-units specified by that locus.

With this possibility in mind it is interesting to compare the occurrence and pattern of complementation at what are almost certainly analogous loci in four microorganisms ; Salmonella typhimurium, Neurospora crassa, Saccharomyces cerevisie, and Schizosaccharomyces pombe. In these organisms mutations at two loci concerned in adenine synthesis result in the accumulation of red pigments. Mutant alleles at one of the two loci in each organism show complementation but not those at the other (see table 2).

Furthermore the maps for $a d-3 B$, $a d-2$ and $a d-6$ are complex. That for $a d-3 B$ is linear (de Serres, personal communication) and has I 7 sub-units (de Serres, 1964), whereas the maps for ad-2 and $a d-6$ both consist of three sections, one linear and two circular (Leupold and Gutz, 1964). On the present hypothesis of complementation these four loci specify polymeric enzymes and probably carry out the same reaction in the organisms concerned. The similarity between the saccharomyces suggests that in these two organisms the enzymes may resemble each other in their tertiary structure.

\section{SUMMARY}

r. One hundred and fifty mutants at the ad-2 locus of Saccharomyces cerevisie have been tested for interallelic complementation. Fifty-six of the mutants failed to complement in any of the tests; of the remainder which did complement fifty-one were non-leaky and forty-three were leaky. 
2. The complementation map for seventy-eight of the complementing mutants comprised three sections, two circular and one linear.

3. There were twenty-three results in which the complementation matrix and map did not correspond.

4. Analysis of the complementation data suggests that leaky mutants complement more frequently than non-leaky mutants.

5. Some correlations between the occurrence of complementation at analogous loci in Salmonella, Neurospora, Saccharomyces and Schizosaccharomyces are discussed.

Acknowledgments.-One of us (R.A.W.) is indebted to the Department of Scientific Research for a Research Studentship, during tenure of which this work was carried out.

\section{REFERENCES}

BEVAN, E. A., AND Woods, R. A. 1962. Complementation between adenine-requiring mutants in yeast. (Abstr.) Heredity, 17, $14 \mathrm{I}$.

BRENNER, s. 1959. The mechanism of gene action. CIBA Foundation Symposium on the Biochemistry of Human Genetics. Churchill, London, pp. 304-32 I.

GALEP, E. 1956. Functional relationships between three adenineless mutants in Aspergillus nidulans. (Abstr.) Heredity, 10, 279.

GARLSON, E. A. 1961. Limitations of geometrical models for complementation mapping of allelic series. Nature, $191,788-790$.

GASE, M. E., AND GILES, N. H. 1960. Comparative complementation and genetic maps of the pan-2 locus in Neurospora crassa. Proc. Natl. Acad. Sci. U.S., 46 , $659-676$.

Gatcheside, D. G., AND overton, A. 1958. Complementation between alleles in heterokaryons. Cold Spring Harbor Symp. Quant. Biol., 23, 137-140.

Costello, W. P., AND BEVAN, E. A. 1964. Complementation between ad-5/7 alleles in yeast. Genetics, 50, $1219-1230$.

Cox, B. S., AND bevan, E. A. I962. Aneuploidy in yeast. New Phytol., 6r, 342-355. CRICK, P. H. C., AND ORGEL, L. E. 1964. The theory of inter-allelic complementation. 7. Mol. Biol., 8, 161-165.

DE SERREs, F. J. 196o. Studies with purple adenine mutants in Neurospora crassa. IV. Lack of complementation between different $a d-3 A$ mutants in heterokaryons and pseudowild types. Genetics, 45, 555-566.

DE SERRES, F. J. 1963. Studies with purple adenine mutants in Neurospora crassa. V. Evidence for allelic complementation among $a d-3 B$ mutants. Genetics, $4^{8}$, $35 \mathrm{I}-36 \mathrm{o}$.

DE SERres, F. J. 1964. Mutagenesis and chromosome structure. F. Cell and Comp. Physiol., 64, (Suppl. I), 33-42.

DORFMAN, B. 1964. Allelic complementation at the ad-5/7 locus in yeast. Genetics, 50,123 I-1 243 .

EMEIS, c. C., AND GUTZ, H. 1958. Eine einefache technik zur massenisolation von hefesporen. Z. Naturforshg, ${ }_{3} 36,647-650$.

EPHRUSSI, B., HotTINGUER, H., AND TAVLITSKI, J. 1949. Action de l'acriflavine sur les levures. II. Etude genetique du mutant " petite colonie". Ann. Inst. Past., $76,419-45^{\circ}$.

Fincham, J. R. S., AND codDington, A. 1963. Complementation at the am locus of Neurospora crassa: a reaction between different mutant forms of glutamate dehydrogenase. 7. Mol. Biol., 6, 361-373.

FINCHAM, J. R. S., AND STADLER, D. R. 1965. Complementation relationships of Neurospora am mutants in relation to their formation of abnormal varieties of glutamate dehydrogenase. Genet. Res., Camb., 6, 121-129. 
GILes, N. H. 1958, Mutations at specific loci in Neurospora. Proc. Ioth Intern. Congr. Genet., $1,261-279$.

GILES, N. H., PARTRIDGE, C. W. H., AND NELSON, R. R. 1957. The genetic control of adenylosuccinase in Neurospora crassa. Proc. Natl. Acad. Sci. U.S., 43, 305-31 7.

GOTS, J. S., COON, R. G., AND HILD, D. H. 1959. Cistronic control of biosynthetic leakage in purine auxotrophs of Salmonella typhimurium. Bact. Proc., G25, 35 .

GUTz, H. 1963. Untersuchungen zur Feinstruktur der Gene $\operatorname{ad}_{7}$ und $\operatorname{ad}_{6}$ von Schizosaccharomyces pombe LIND. Habilitationsschrift. Techinschen Universität, Berlin.

IsHIKAWA, T. 1960. Complementation and genetic maps of the ad-8 locus in Neurospora crassa. (Abstr.) Genetics, 45, 993.

JOHNSTON, J. R., AND MORTIMER, K. R. 1959. Use of snail digestive juice in isolation of yeast spore tetrads. F. Bact., $78,292$.

KAPUleR, A. M., AND BERNsrein, H. 1963. A molecular model for an enzyme based on a correlation between the genetic and complementation maps of the locus specifying the enzyme. 7. Mol. Biol., 6, 443-45I.

KEMP, R. F. O., AND BEVAN, E. A. 1959. New techniques for isolating spores of Hymenomycetes. Trans. Brit. Mycol. Soc., 42, 308-311.

LEDERBERG, J., AND LEDERBERG, E. M. 1952. Replica plating and indirect selection of bacterial mutants. 7. Bact., $63,399-406$.

LEUPOLD, U. 1961. Intragene Rekombination und allele Komplementierung. Arch. Fulius-Klaus-Stifting, 36, 89-1 17.

LEUPOLD, U., AND GUTZ, H. 1964. Genetic fine structure in Schizosaccharomyces. Genetics Today. Proc. XIth, Intern. Cong. Genet., I, 31-35.

LEVINTHAL, M., FOGEL, S., AND HURST, D. D. 1962. Genetic and biochemical analysis of purine biosynthetic pathways in Saccharomyces. (Abstr.). Genetics, 47, 967.

LEVINTHAL, C., SIGNER, E. R., AND FETHEROLF, K. 1962. Reactivation and hybridisation of alkaline phosphatase of Escherichia coli KI2. Proc. Natl. Acad. Sci. U.S., $48,1230-1237$.

MEGNET, R. 1958. Complementation between adenylosuccinaseless mutants at the ad-8 locus of Schizosaccharomyces pombe. (Abstr.) Genetics, 44, 526.

REAUME, S. E., AND TATUM, E. L. 1949. Spontaneous and nitrogen mustard induced nutritional deficiencies in Saccharomyces cerevisea. Arch. Biochem., 22, 331-338.

ROTHMAN, R., AND BYRNE, R. 1963. Fingerprint analysis of alkaline phosphatase of Escherichia coli K12. 7. Mol. Biol., 6, 330-340.

SCHLESINGER, M. J., AND LEVINTHAL, c. 1963. Hybrid protein formation of $E$. coli alkaline phosphatase leading to in vitro complementation. F. Mol. Biol., 7, 1-2. 